\title{
Comparison of strain imaging techniques in CRT candidates: CMR tagging, CMR feature tracking and speckle tracking echocardiography
}

\author{
Wouter M. van Everdingen ${ }^{1} \cdot$ Alwin Zweerink $^{2} \cdot$ Robin Nijveldt $^{2} \cdot$ Odette A. E. Salden $^{1} \cdot$ Mathias Meine $^{1}$ • \\ Alexander H. Maass ${ }^{3}$ - Kevin Vernooy ${ }^{4}$ Frederik J. De Lange ${ }^{5}$ Albert C. van Rossum - Pierre Croisille $^{6}$.

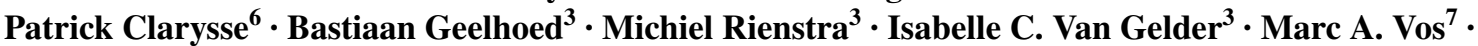 \\ Cornelis P. Allaart ${ }^{2}$ - Maarten J. Cramer ${ }^{1}$
}

Received: 14 August 2017 / Accepted: 29 September 2017 / Published online: 17 October 2017

(C) The Author(s) 2017. This article is an open access publication

\begin{abstract}
Parameters using myocardial strain analysis may predict response to cardiac resynchronization therapy (CRT). As the agreement between currently available strain imaging modalities is unknown, three different modalities were compared. Twenty-seven CRT-candidates, prospectively included in the MARC study, underwent cardiac magnetic resonance (CMR) imaging and echocardiographic examination. Left ventricular (LV) circumferential strain was analysed with CMR tagging (CMR-TAG), CMR feature tracking (CMR-FT), and speckle tracking echocardiography (STE). Basic strain values and parameters of dyssynchrony
\end{abstract}

Wouter M. van Everdingen, Alwin Zweerink, Cornelis P.

Allaart and Maarten J. Cramer have contributed equally to the manuscript.

Electronic supplementary material The online version of this article (doi:10.1007/s10554-017-1253-5) contains supplementary material, which is available to authorized users. and discoordination obtained with CMR-FT and STE were compared to CMR-TAG. Agreement of CMR-FT and CMRTAG was overall fair, while agreement between STE and CMR-TAG was often poor. For both comparisons, agreement on discoordination parameters was highest, followed by dyssynchrony and basic strain parameters. For discoordination parameters, agreement on systolic stretch index was highest, with fair intra-class correlation coefficients (ICC) (CMR-FT: 0.58, STE: 0.55). ICC of septal systolic rebound stretch $\left(\mathrm{SRS}_{\text {sept }}\right)$ was poor (CMR-FT: 0.41, STE: 0.30). Internal stretch factor of septal and lateral wall (ISF sep-lat $)$ showed fair ICC values (CMR-FT: 0.53, STE: 0.46), while the ICC of the total LV $\left(\mathrm{ISF}_{\mathrm{LV}}\right)$ was fair for CMR-FT (0.55) and poor for STE (ICC: 0.32). The CURE index had a fair ICC for both comparisons (CMR-FT: 0.49, STE 0.41). Although comparison of STE to CMR-TAG was limited by methodological differences, agreement between CMR-FT and CMRTAG was overall higher compared to STE and CMR-TAG.

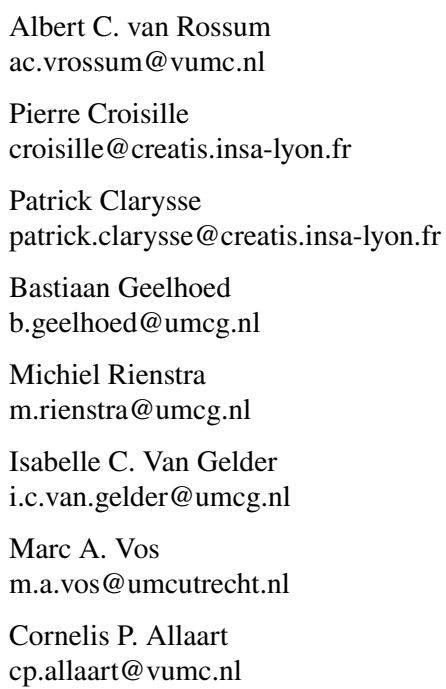

Cornelis P. Allaart

cp.allaart@vumc.nl 
CMR-FT is a potential clinical alternative for CMR-TAG and STE, especially in the detection of discoordination in CRT-candidates.

Keywords Strain $\cdot$ Myocardial tagging $\cdot$ Feature tracking $\cdot$ Speckle tracking echocardiography .

Dyssynchrony $\cdot$ Discoordination $\cdot$ Cardiac

resynchronization therapy

\begin{tabular}{|c|c|}
\hline \multicolumn{2}{|c|}{ Abbreviations } \\
\hline AVC strain & Strain value at aortic valve closure \\
\hline AVC & Aortic valve closure \\
\hline CMR & Cardiac magnetic resonance imaging \\
\hline CMR-FT & Cardiac magnetic resonance feature tracking \\
\hline CMR-TAG & $\begin{array}{l}\text { Cardiac magnetic resonance myocardial } \\
\text { tagging }\end{array}$ \\
\hline CRT & Cardiac resynchronization therapy \\
\hline CSPAMM & $\begin{array}{l}\text { Complementary spatial modulation of } \\
\text { magnetization }\end{array}$ \\
\hline CURE & Circumferential uniformity ratio estimates. \\
\hline ICC & Intra-class correlation coefficient \\
\hline $\mathrm{ISF}_{\mathrm{LV}}$ & $\begin{array}{l}\text { Internal stretch factor of all left ventricular } \\
\text { segments }\end{array}$ \\
\hline ISF $_{\text {sep-lat }}$ & $\begin{array}{l}\text { Internal stretch factor of septum and lateral } \\
\text { wall }\end{array}$ \\
\hline LBBB & Left bundle branch block \\
\hline LV & Left ventricle \\
\hline LVEDV & Left ventricular end-diastolic volume \\
\hline LVEF & Left ventricular ejection fraction \\
\hline LVESV & Left ventricular end-systolic volume \\
\hline MARC & $\begin{array}{l}\text { Markers of response to cardiac resynchroni- } \\
\text { zation therapy }\end{array}$ \\
\hline NYHA & New York Heart Association \\
\hline
\end{tabular}

Maarten J. Cramer

m.j.m.cramer@umcutrecht.nl

1 Department of Cardiology, University Medical Centre Utrecht, Utrecht, The Netherlands

2 Department of Cardiology, and Institute for Cardiovascular Research (ICaR-VU), VU University Medical Centre, Amsterdam, The Netherlands

3 Department of Cardiology, Thoraxcenter, University of Groningen, University Medical Centre Groningen, Groningen, The Netherlands

4 Department of Cardiology, Maastricht University Medical Centre, Maastricht, The Netherlands

5 Department of Cardiology, Academic Medical Centre, Amsterdam, The Netherlands

6 Université Lyon, UJM-Saint-Etienne, INSA, CNRS UMR 5520, INSERM U1206, CREATIS, 42023 Saint-Etienne, France

7 Department of Medical Physiology, University of Utrecht, Utrecht, The Netherlands
Onset-delay Time delay between onset of shortening of septal and lateral wall

Peak-delay Septal to lateral wall delay of time to maximal peak shortening

$\mathrm{R} \quad$ Correlation coefficient

RVEF Right ventricular ejection fraction.

SRS $_{\text {sept }} \quad$ Septal systolic rebound stretch

SSFP Steady-state free-precession

SSI Systolic stretch index

STE Speckle tracking echocardiography

TE Echo time

TR Repetition time

TTP $_{\max } \quad$ Time to maximal peak shortening

TTP $_{\mathrm{SD}} \quad$ Standard deviation if time to peak max of all segments

\section{Introduction}

Cardiac resynchronization therapy (CRT) is an established treatment for patients with heart failure, reduced left ventricular (LV) ejection fraction, and a prolonged QRS caused by a left bundle branch block (LBBB) or nonspecific intraventricular conduction delay [1]. CRT aims to restore LV mechanics and improve hemodynamic by resynchronization of LV electrical activation [2]. Unfortunately, the effect of CRT is limited in $30-40 \%$ of the patients, partly due to a lack of optimal criteria for patient selection [3, 4]. In current international guidelines the selection criteria for CRT are limited to clinical parameters, ECG parameters and LV ejection fraction $\leq 35 \%$ [1]. Patient selection for CRT may be improved with additional parameters reflecting mechanical dyssynchrony or discoordination obtained with strain analysis on imaging [4-7]. These parameters reflect the LV mechanical consequences caused by an inhomogeneous electrical activation. Mechanical dyssynchrony parameters are based on timing differences between particular LV segments $[8,9]$. However, these mechanical dyssynchrony parameters showed disappointing results in large multi-centre trials [9]. More promising parameters focus on discoordination, reflecting a percentage or fraction of opposing (i.e. inefficient) deformation [6,10-12]. These parameters are determined using myocardial strain analysis, which can be obtained with several cardiac imaging techniques, including cardiac magnetic resonance imaging (CMR) with tagging (CMR-TAG), CMR cine images and a post-processing technique named feature tracking (CMR-FT), and speckle tracking echocardiography (STE) [13, 14]. Although CMRTAG is regarded as the non-invasive 'gold-standard', it is generally limited to scientific applications, requiring specific imaging protocols, sequences, and dedicated post-processing software. Clinical application of CMR-FT and STE is more feasible compared to CMR-TAG, as both techniques 
are applicable to images obtained during standard clinical imaging protocols [14-16]. Nevertheless, both techniques (i.e. CMR-FT and STE) lack validation on strain parameters reflecting mechanical dyssynchrony and discoordination. Thus, no study has yet compared results obtained with all three techniques (i.e. CMR-TAG, CMR-FT and STE) in patients eligible for CRT. This study aims to compare circumferential strain parameters obtained with CMR-FT and STE versus gold-standard CMR-TAG in patients eligible for CRT. The comparison of indices reflecting mechanical dyssynchrony and discoordination are of specific interest.

\section{Materials and methods}

This sub study is part of the Markers of Acute Response to CRT (MARC) study (Cohfar, CTMM, The Netherlands, clinicaltrials.gov: NCT01519908), which was designed to investigate predictors for response on CRT. The MARC study included 240 patients planned for CRT implantation in six medical centres in the Netherlands, using previously published in- and exclusion criteria [17]. Twenty-seven of the 240 patients were included in this sub-study, as these patients gave consent for an additional CMR examination including myocardial tagging in the VU university medical centre (Amsterdam, The Netherlands). All subjects gave written informed consent and the local medical ethics committees approved data collection and management. The investigation conforms to the principles outlined in the Declaration of Helsinki.

\section{Echocardiographic examination}

Echocardiographic examinations were performed on either GE Vivid7, GE Vivid9 (General Electric Healthcare, Chicago, Illinois, USA), or Philips iE33 (Philips Medical Systems, Best, The Netherlands) ultrasound machines prior to CRT implantation by all participating centres and analysed by the echocardiographic core lab (WE and MC, UMC Utrecht, Utrecht, The Netherlands).

\section{Acquisition—standard echocardiographic images}

Standard echocardiographic images were obtained, including a parasternal short axis view at the papillary muscle and at the mitral valve level [18]. Image quality and frame rate $(50-100 \mathrm{~Hz})$ were optimized for offline speckle tracking analysis. Pulsed-wave Doppler images of the mitral valve inlet and LV outflow tract were obtained of mitral valve and aortic valve closure (AVC) to define systole.

\section{Offine analysis—speckle tracking echocardiography}

Echocardiographic images were exported as DICOM-files for vendor-independent strain analysis (TomTec 2D Cardiac Performance Analysis (2DCPA) version 1.2.1.2, TomTec Imaging Systems, Unterschleissheim, Germany). A region of interest was placed by user defined markers at the endocardial border. The epicardial border was excluded, as it often lacked a clear border zone. The region of interest was automatically separated into six segments. Segments were excluded if, even after repeated adjustment of the region of interest, adequate tracking was not achievable. The marker for reference length was placed at QRS onset.

STE results were exported for analysis with author written scripts for Matlab 2014b (Mathworks, Natick, MA, USA). Segmental strain curves were discarded in case of low signal-to-noise ratio as judged by two independent investigators (WE and AZ). At least two segments needed to be analysable per wall. Results of strain parameters of the septum were based on averages of maximal four septal segments (i.e. basal- and mid-level of inferoseptal and anteroseptal segments) while the lateral wall parameters were based on averages of maximal four lateral wall segments (i.e. basal- and mid-level of inferolateral and anterolateral segments). The post-processing and selection of analysable segments and averaging into one septal and one lateral wall strain curve, was similar for STE, CMR-TAG and CMR-FT.

\section{Cardiac magnetic resonance imaging}

CMR examinations were performed on a $1.5 \mathrm{~T}$ system (Magnetom Avanto, Siemens, Erlangen, Germany) with the use of a phased array cardiac receiver coil. Although performed on a different moment compared to STE, both standard CMR cine images for the CMR-FT analysis and CMR tagging images were obtained in the same examination.

\section{Acquisition-standard CMR images}

Standard CMR cine images were acquired using a retrospectively ECG-gated balanced steady-state free-precession (SSFP) sequence during end-expiratory breath holding. A stack of 8-12 consecutive short axis cine images was acquired covering the entire LV. Typical image acquisition parameters were: slice thickness $5 \mathrm{~mm}$, slice gap $5 \mathrm{~mm}$, echo time (TE) $1.6 \mathrm{~ms}$, repetition time (TR) $3.2 \mathrm{~ms}$, temporal resolution $<50 \mathrm{~ms}$, in-plane spatial resolution 1.5 by $2.1 \mathrm{~mm}$, flip angle $60^{\circ}$. The number of reconstructed temporal phases within the cardiac cycle was set at 20. Subsequently, high temporal resolution (TE $1.7 \mathrm{~ms}$, TR $3.4 \mathrm{~ms}$, temporal resolution $\sim 15 \mathrm{~ms}$ ) cine imaging of the LV in the three-chamber view was performed to assess the opening and closure times of the mitral and aortic valve. 
Acquisition-CMR tagging images

Before contrast injection, tagged images were acquired at three short-axis slices (basal, mid, apical) using a complementary spatial modulation of magnetization (CSPAMM) line tagging sequence with segmented ECG-gated acquisitions and serial breath holds [16]. Typical image acquisition parameters were: slice thickness $6 \mathrm{~mm}$, TE $1.7 \mathrm{~ms}$, TR $3.6 \mathrm{~ms}$, temporal resolution $<15 \mathrm{~ms}$, in-plane spatial resolution 1.3 by $4.3 \mathrm{~mm}$, flip angle $20^{\circ}$, tag spacing $7 \mathrm{~mm}$. The number of reconstructed temporal phases within the cardiac cycle was set at 55 .

\section{Offline analysis-CMR tagging}

Tagged CMR images were exported and analysed (AZ, RN) with the SinMod technique (inTag, v2.0, CREATIS lab, Lyon, France, run as a plug-in for OsiriX Imaging Software v6.5, Pixmeo, Switzerland) [19]. Apical slices were discarded, while basal and mid short-axis slices were used for analysis in order to match the STE slice positions. After selecting the area of interest, endocardial and epicardial contours were manually drawn in the end-systolic phase and automatically propagated. A template was placed dividing the LV in six equally sized regions, similar to STE. The myocardium was divided in three layers (i.e. endo-, mid-, epi-wall layer). Results of the mid-wall layer were used, as these results are independent of contour placement.

\section{Offline analysis-CMR feature tracking}

Semi-automated FT analysis software (QStrain Research Edition evaluation version 1.3.0.10, Medis, Leiden, The Netherlands) was used to analyse short-axis cine images corresponding with the mid and basal slice-location of the CMR-TAG images (AZ and RN). Apical slices were discarded to match STE. First, endo- and epicardial contours were manually drawn in both end-diastolic and end-systolic frames and propagated automatically. Both endocardial and epicardial features were included for strain analysis, resulting in myocardial strain. The $\mathrm{LV}$ was divided in six regions, similar to the other techniques.

\section{Basic strain parameters}

The following parameters were obtained for the septal and lateral wall (Fig. 1). (1) Peak strain was the maximal negative peak strain during the cardiac cycle. (2) AVC strain was defined as the strain value at aortic valve closure. (3) Time to maximal peak $\left(\mathrm{TTP}_{\max }\right)$ was the time difference between the start of the strain curve to most negative peak strain. Furthermore, (4) average systolic strain rate (i.e. average strain rate between mitral valve closure and AVC) and (5) average diastolic strain rate (i.e. average strain rate after AVC) were obtained.

\section{Dyssynchrony parameters}

Three parameters of dyssynchrony were analysed. (a) Onsetdelay was determined as the absolute time delay between onset of shortening of the septal and lateral wall. (b) Peak delay was calculated as the absolute difference between lateral and septal wall $\mathrm{TTP}_{\max }$. (c) The $\mathrm{TTP}_{\mathrm{SD}}$ was calculated as the standard deviation of $\mathrm{TTP}_{\max }$ of all analysable segments of the total LV.

\section{Regional discoordination parameters}

Three regional discoordination parameters were analysed. (d) Systolic rebound stretch of the septum $\left(\mathrm{SRS}_{\text {sept }}\right)$ was defined as the total amount of systolic stretch after initial shortening of the septum (Fig. 1). (e) Systolic stretch index (SSI) was calculated by adding SRS $_{\text {sept }}$ to all systolic stretch of the lateral wall [11]. (f) Internal stretch factor (ISF) was calculated as the fraction of all systolic stretch compared to cumulative systolic shortening for the septal and lateral wall $\left(\mathrm{ISF}_{\text {sep-lat }}\right)$. (g) Septal strain curves were categorized in three types, determined by their shape, LBBB-1: doublepeaked systolic stretch, LBBB-2: early pre-ejection shortening peak followed by prominent systolic stretching and LBBB-3: pseudo normal shortening with a late-systolic shortening peak, followed by less pronounced end-systolic stretch (Fig. 2) [12].

\section{Discoordination parameters of the total LV}

Finally, two discoordination parameters reflecting the total LV were analysed. (h) The internal stretch factor of the total LV ( ISF $_{\mathrm{LV}}$ ) was determined using all analysable segments. $\mathrm{ISF}_{\mathrm{LV}}$ was determined as the total amount of stretch divided by the total amount of shortening during systole (supplemental Fig. 1) [20]. (i) Lastly, the circumferential uniformity ratio estimates (CURE) was calculated, ranging from 0 (i.e. total dyssynchrony) to 1 (i.e. perfectly synchronous) [21].

\section{Statistical analysis}

Statistical analysis was performed (BG and $\mathrm{MR}$ ) using $\mathrm{R}$ version 3.3.2 (The $\mathrm{R}$ foundation for Statistical Computing), and the R-packages psych version 1.5 .8 (for calculation of Cohen's kappa coefficients, ICCs and their associated p values). Results obtained with the three techniques were compared using the intra-class correlation coefficient (ICC) for absolute agreement between techniques (ICC2 according to Shrout and Fleiss) [22] and Spearman rank or Pearson 

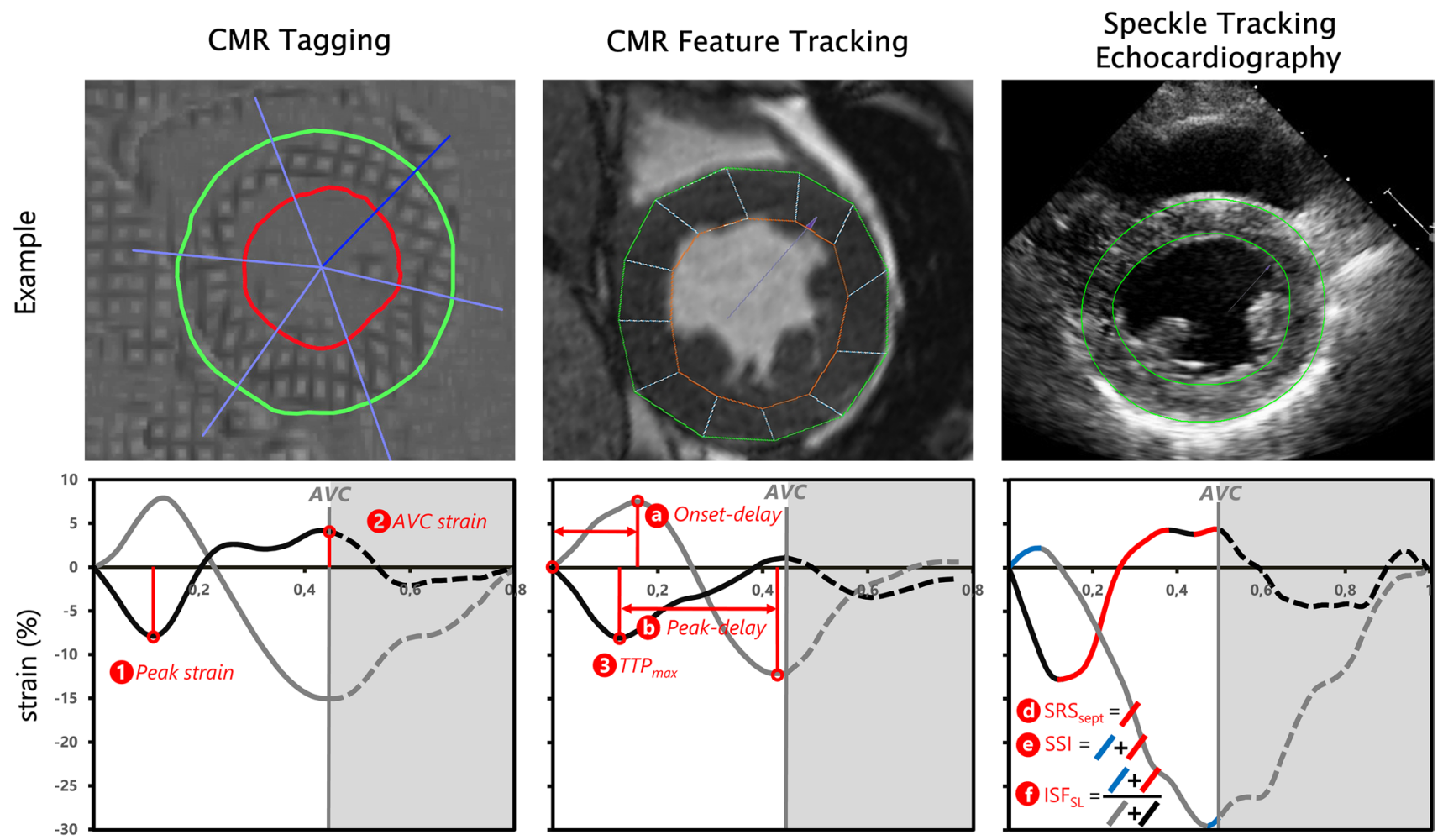

time (s)

Fig. 1 Overview of imaging techniques and corresponding myocardial strain analysis. Examples of imaging techniques (top row) and resulting strain signals (bottom row) of one specific patient. Each column represents a single technique with the corresponding strain results. Examples of derived parameters are shown per graph. Basic strain parameters are indicated with a number, dyssynchrony and discoordination parameters are indicated with a character. Strain signals of the septum (black line) and lateral wall (grey line) are given, with

correlation coefficient $(\mathrm{R})$ depending on normality of data. An ICC $\geq 0.75$ was classified as excellent, $0.60-0.74$ as good, $0.40-0.59$ as fair, and $<0.40$ as poor [23]. Bland-Altman plots were made to observe the agreement between modalities. The mean difference and limits of agreement ( \pm 1.96 standard deviation) of the Bland-Altman plot were used a reference of agreement. Lastly, Cohen's kappa coefficient was calculated as the level of agreement between modalities on septal strain pattern categorization. A statistical result with a $p$ value $<0.05$ was deemed significant.

\section{Results}

\section{Study population}

Twenty-seven patients with CMR tagging images were included, of which a detailed description is given in Table 1 . In these patients $94 \%$ of all segments were analysable with CMR-TAG, $87 \%$ with CMR-FT and $89 \%$ with STE. Frame the aortic valve closure (grey vertical line) as end of systole. CMR cardiac magnetic resonance imaging, $A V C$ aortic valve closure, $A V C$ strain strain value at aortic valve closure, $T T P_{\max }$ time to maximal peak shortening, onset-delay time delay between onset of shortening of septal and lateral wall, peak-delay septal to lateral wall delay of $\mathrm{TTP}_{\max }, S R S_{\text {sept }}$ systolic rebound stretch of the septum, SSI systolic stretch index, $I S F_{\text {sep-lat }}$ internal stretch fraction of septal and lateral wall

rate of echocardiographic images was on average $65 \pm 11 \mathrm{~Hz}$, which corresponds to a temporal resolution of $\sim 15 \mathrm{~ms}$. Temporal resolution of CMR-TAG was $\sim 14 \mathrm{~ms}$, while it was $\sim 40 \mathrm{~ms}$ for CMR-FT.

\section{Basic strain parameters}

Overall, agreement of CMR-TAG and CMR-FT was higher compared to agreement of CMR-TAG and STE for basic strain parameters. This applied for ICC values, Bland-Altman characteristics and the correlation coefficient (R) (Table 3). (1) For CMR-FT AVC strain of the septum was fair (ICC 0.55, R 0.67), while it was poor for STE (ICC 0.23 , R 0.47). The ICC of AVC strain of the lateral wall was fair for CMR-FT (ICC 0.50, R 0.50) and poor for STE (ICC 0.08, R 0.10). (2) Peak strain of the septum had a fair ICC for CMR-FT (ICC 0.58, R 0.55) and a poor ICC for STE (ICC 0.155, 0.42). Lateral wall peak strain also had a fair ICC for CMR-FT (ICC 0.54, R 0.59) and a poor ICC 


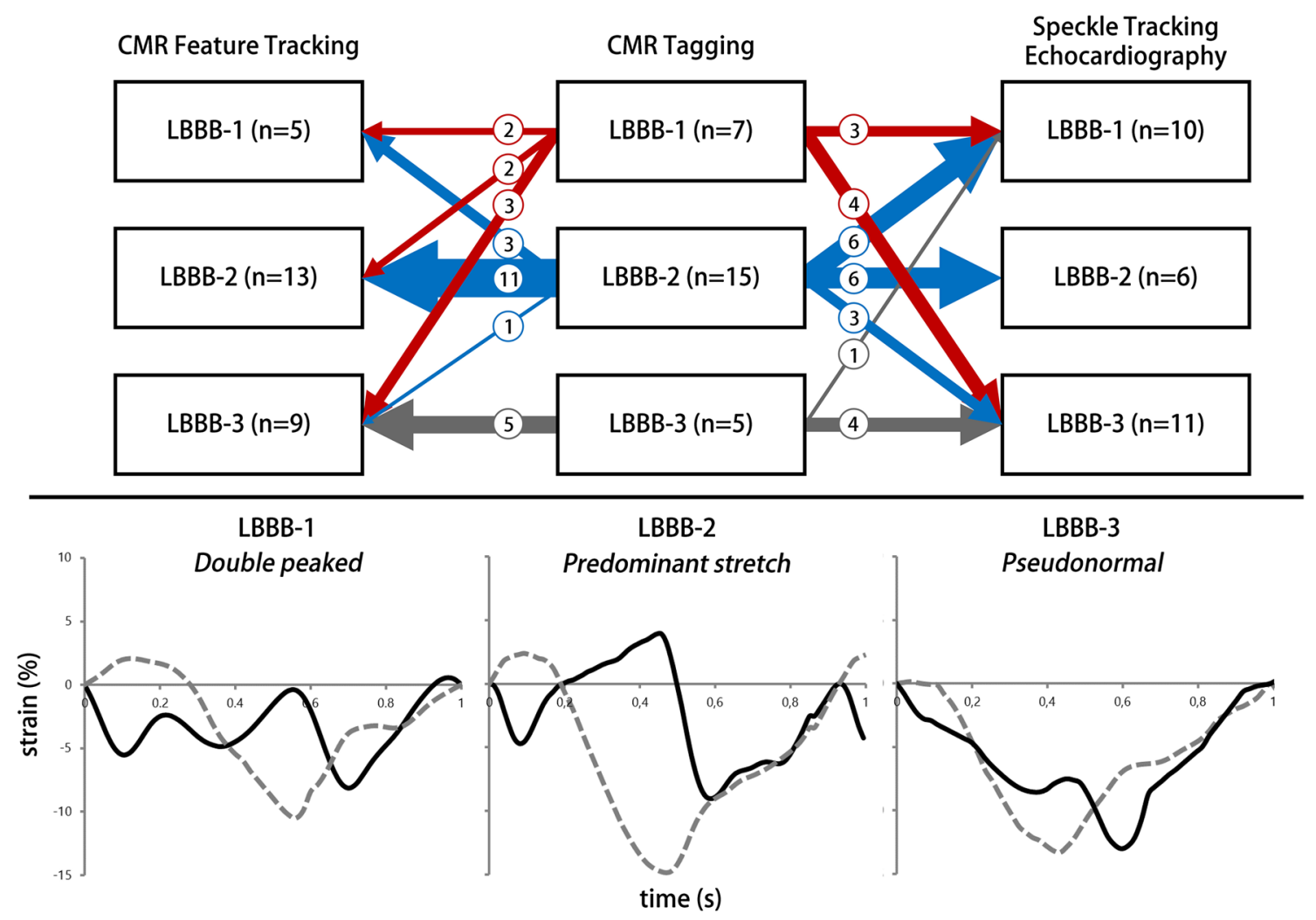

Fig. 2 LBBB pattern categorization. Septal strain pattern categorization and distribution of strain patterns found by the three imaging techniques. The distribution per imaging technique is given vertical in the upper panel. The cross-over of patients from CMR tagging to speckle tracking echocardiography and CMR feature tracking is displayed by arrows. The thickness of the arrows matches the number of patients crossing over. The number of patients crossing over is also given by a number in each arrow. Specific examples of the three patterns are given in the lower panel. Black curve: septal strain, grey dashed curved: lateral wall strain. $C M R$ cardiac magnetic resonance imaging, $L B B B-1$ double peak shortening, $L B B B-2$ predominant stretch, $L B B B-3$ pseudo-normal shortening, $n$ number of patients for STE (ICC 0.01, R 0.02). (3) $\mathrm{TTP}_{\max }$ of the septal and lateral wall showed an apparent wide distribution in the Bland-Altman plots for both comparisons (Fig. 3). Septal $\mathrm{TTP}_{\text {max }}$ caused a large spread in results, while the lateral wall TTP $_{\text {max }}$ were more similar for both comparisons. Although ICC for $\mathrm{TTP}_{\max }$ was poor for all comparisons, CMR-FT showed better agreement with CMR-TAG compared to STE for the septum (CMR-FT: ICC 0.17, R 0.11 and STE: ICC 0.00, R -0.16) and the lateral wall (CMRFT: ICC 0.34, R 0.40 and STE: ICC 0.13, R 0.23). (4) Systolic strain rate showed comparable results to AVC strain. For CMR-FT systolic strain rate of the septum was fair (ICC 0.56, R 0.66), while it was poor for STE (ICC 0.25, $\mathrm{R} 0.45)$. ICC of the systolic strain rate of the lateral wall was fair for CMR-FT (ICC 0.575, R 0.58) and poor for STE (ICC 0.05, R 0.05). (5) Diastolic strain rate showed good ICC for the septal (ICC 0.64, R 0.66) and excellent ICC for the lateral wall (ICC 0.82, 0.82) for CMR-TAG versus CMR-FT. ICC of diastolic strain rate was poor for both walls comparing CMR-TAG and STE (septum: ICC 0.34, R 0.50, lateral wall: ICC 0.23, R 0.38).

\section{Dyssynchrony parameters}

(a) Onset delay was quite similar for CMR-TAG and CMRFT, with a mean difference in the Bland-Altman plot of -2.5 ms (Supplemental Fig. 2). The corresponding ICC was fair (ICC 0.42, R 0.23). CMR-TAG versus STE also had a low mean difference of $-1.9 \mathrm{~ms}$, although the limits of agreement where larger, combined with a poor ICC (ICC 0.024, R -0.08). (b) Peak delay of CMR-TAG was overall larger compared to CMR-FT and STE (Supplemental Fig. 2). ICC was fair for CMR-FT (ICC 0.45, R 0.46), and poor for STE (ICC 0.23, R 0.27). TTP ${ }_{\mathrm{SD}}$ (c) showed a fair ICC for CMR-FT (ICC 0.46, R 0.49), and poor ICC for STE (ICC 0.20 , R 0.19). 
Table 1 Baseline characteristics

\begin{tabular}{ll}
\hline Variable & Total cohort $(\mathrm{n}=27)$ \\
\hline Age (years) & $65.1 \pm 9.7$ \\
Gender (n, male) & $15(56 \%)$ \\
Aetiology (n, ischemic cardiomyopathy) & $7(26 \%)$ \\
BMI (kg/m²) & $26.3 \pm 3.9$ \\
QRS width (ms) & $183(167-194)$ \\
Sinus rhythm (\%) & $100 \%$ \\
QRS morphology (n) & \\
LBBB & $21(81 \%)$ \\
IVCD & $6(19 \%)$ \\
NYHA class (n) & \\
II & $17(63 \%)$ \\
III & $10(37 \%)$ \\
Medication (n) & \\
Beta-blockers & $23(85 \%)$ \\
Diuretics & $22(81 \%)$ \\
ACE/ATII inhibitors & $17(63 \%)$ \\
Aldosterone antagonists & $10(37 \%)$ \\
CMR-LVEDV (ml) & $317 \pm 100$ \\
CMR-LVESV (ml) & $239 \pm 99$ \\
CMR-LVEF (\%) & $26.7 \pm 8.8$ \\
CMR-LV mass (gr) & $131(118-157)$ \\
\hline
\end{tabular}

Mean and standard deviation are given with \pm symbol, median and interquartile range between brackets

$B M I$ body surface mass index, $C M R$ cardiac magnetic resonance imaging, $L B B B$ left bundle branch block, IVCD intraventricular conduction delay, NYHA New York Heart Association, ATII angiotensin receptor II, $L V E D V$ left ventricular end-diastolic volume, $L V E S V$ left ventricular end-systolic volume, $L V E F$ left ventricular ejection fraction, $L V$ left ventricular

\section{Regional discoordination parameters}

(d) $\mathrm{SRS}_{\text {sept }}$ showed a fair ICC for CMR-FT (ICC 0.41, R 0.65 ), while agreement was poor for STE (ICC 0.30, R 0.41). CMR-TAG showed overall higher values for $\mathrm{SRS}_{\text {sept }}$ compared to both other imaging techniques. The difference of CMR-TAG to CMR-FT and STE were mostly positive, indicating an underestimation by CMR-FT and STE (Fig. 4). (e) SSI also showed an overall underestimation by CMR-FT and STE compared to CMR-TAG. Agreement on SSI was fair for CMR-FT (ICC 0.58, R 0.68) and STE (ICC 0.55, R 0.70). (f) $\mathrm{ISF}_{\text {sep-lat }}$ was comparable between techniques, ICC's of both CMR-FT (ICC 0.53, R 0.45) and STE (ICC 0.46, R 0.69) were fair. Overall values were still lower by CMR-FT and STE compared to CMR-TAG (Fig. 4). For septal strain patterns (g) the kappa value of CMR-TAG versus CMR-FT $(0.465 \mathrm{p}<0.001)$ was higher compared to the kappa of CMR-TAG versus STE $(0.265$, $\mathrm{p}<0.001)$. The number of patients crossing over from LBBB- 1 or LBBB- 2 on the one hand, and LBBB-3 on the other, using CMR-TAG and CMR-FT is rather low $(n=4$, $15 \%)$, especially compared to CMR-TAG and STE $(n=8$, $30 \%$ ) (Fig. 2).

\section{Discoordination parameters of the total $\mathrm{LV}$}

ICC of $\operatorname{ISF}_{\mathrm{LV}}$ (h) of CMR-FT (ICC 0.55, R 0.66) was the highest off all dyssynchrony and discoordination parameters. Both ICC and R values were lower for STE (ICC 0.32, R 0.42). The CURE index (i) showed rather comparable values between techniques (Fig. 5) with relative narrow limits of agreement in the Bland-Altman plot. Both CMR-FT (ICC 0.485, R 0.37) and STE (ICC 0.41, R 0.36) resulted in a fair ICC value for CURE compared to CMR-TAG (Table 3).

\section{Discussion}

This study explores the comparison of strain parameters in CRT candidates of two widely available strain analysis techniques, speckle tracking echocardiography and CMR feature tracking, with gold-standard CMR tagging. While most basic strain and dyssynchrony parameters differed substantially between techniques, there were apparent similarities found for discoordination parameters. This finding is promising, as discoordination parameters are potential predictors for CRT response [10, 11, 20]. The CMR-based techniques (i.e. CMR-TAG and CMR-FT) showed the highest agreement, shown in fair ICC values, higher $\mathrm{R}$ values, and relative narrow limits of agreement of the Bland-Altman plots. STE mostly had a poor agreement with CMR-TAG. CMR-FT may therefore be a valuable alternative to CMR-TAG for analysis of discoordination parameters in patients eligible for CRT.

\section{Comparison of imaging techniques}

To the best of our knowledge, this is the first study to compare strain parameters between different strain analysis techniques in a CRT patient population. The overall agreement between CMR-FT and CMR-TAG was higher compared to the agreement between STE and CMR-TAG. We would like to discuss three considerations to ascribe this difference. Firstly, STE uses a different imaging source, while both CMR-FT and CMR-TAG are obtained with the same imaging modality. Second, as part of the protocol, echocardiographic examinations and CMR scans were not performed on the same day. Therefore, physiological differences, such as loading conditions and heart rate, may have interfered with agreement of STE and CMR-TAG. Third, the imaging plane used for CMR and echocardiography is possibly 
CMR-TAG vs. CMR-FT
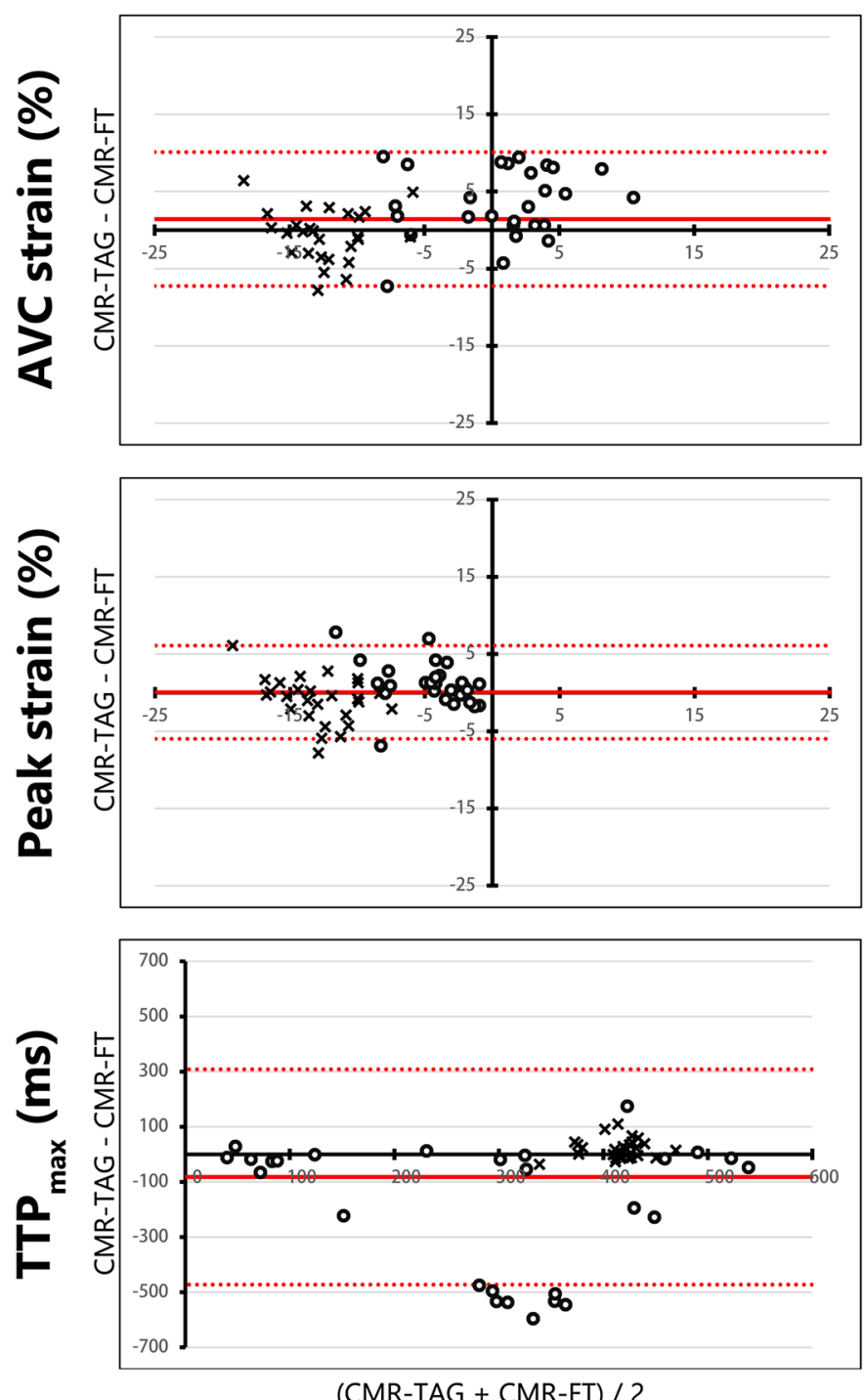

$(C M R-T A G+C M R-F T) / 2$

Fig. 3 Bland-Altman plots of basic strain parameters. Bland-Altman plots for CMR-TAG versus CMR-FT and CMR-TAG versus STE of three basic strain parameters. The mean of two techniques is plotted on the $\mathrm{x}$-axis and the difference on the $\mathrm{y}$-axis. The mean difference is displayed as a solid red line, while the limits of agreement are displayed as dotted red lines. Septal values are given as dots, while lat-

different. Echocardiographic parasternal short-axis views were obtained from a single intercostal position, angulating the echo probe to the mitral valve annulus plane and the papillary muscle plane. These imaging planes may thereby be partly oblique, while CMR imaging planes were 'true' shortaxis views. Furthermore, CMR-FT and CMR-TAG images were acquired on the almost exact same slice position, while the anatomical plane of STE images may be different. Another factor causing discrepancies between techniques is the specific manufacturer used for strain analysis with either CMR-TAG, CMR-FT, or STE [24]. Results of CMR-TAG,

\section{CMR-TAG vs. STE}
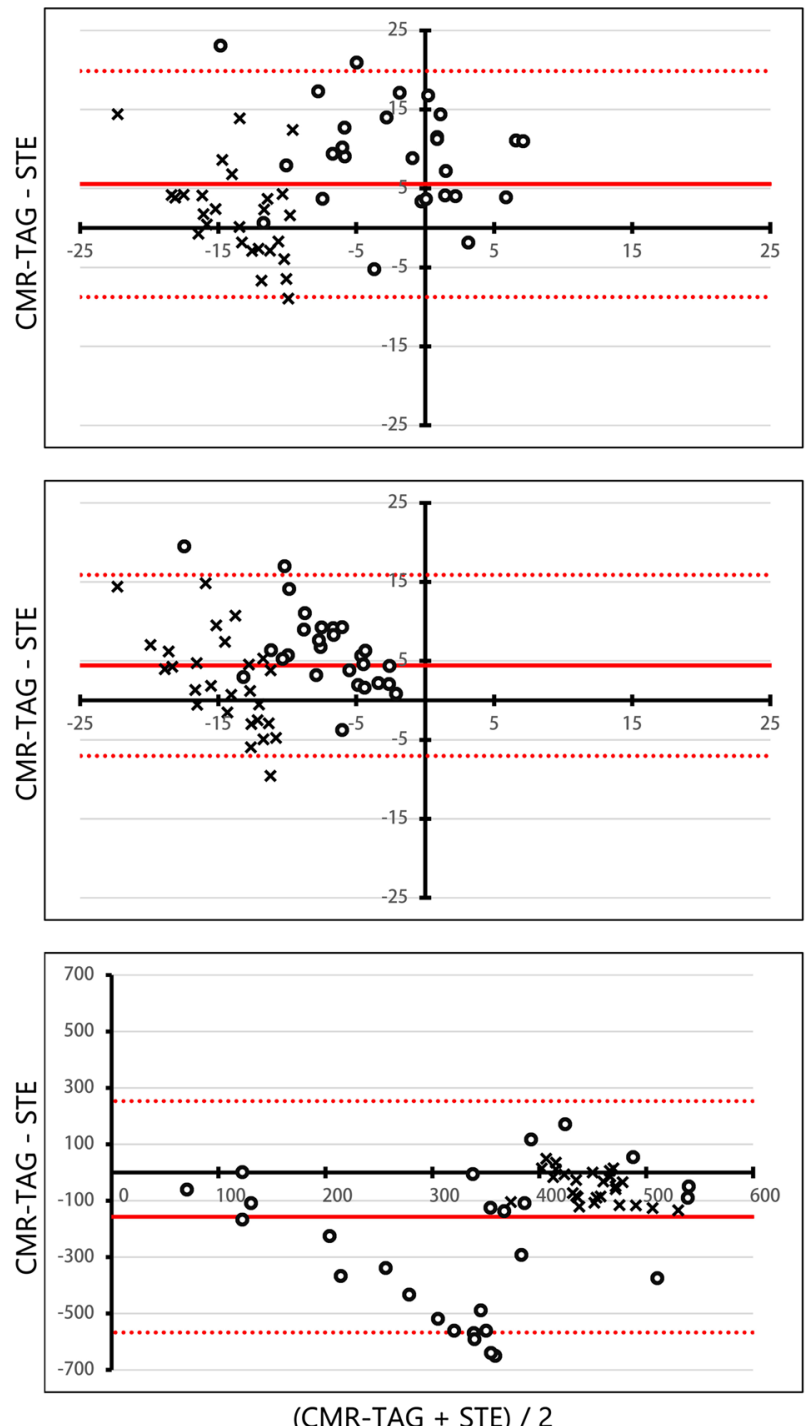

eral wall values are given as crosses. AVC strain strain at aortic valve closure time, Peak strain highest negative peak strain value, $T T P_{\max }$ time to maximal peak strain, $C M R$ cardiac magnetic resonance imaging, TAG tagging, FT feature tracking, STE speckle tracking echocardiography

CMR-FT, and STE are contemporary, as they are dependent on specific analysis algorithms which are constantly under development. Although earlier studies show less favourable agreement of CMR-TAG and CMR-FT, $[25,26]$ recent developments are more promising [27, 28]. This trend is in accordance with our results, as we found that CMRFT had fair agreement with CMR-TAG. However, further improvements are necessary, as results obtained with different imaging techniques can still differ largely for the individual patient. These differences may have underestimated the agreement between STE and CMR-TAG, compared to 
Table 2 Strain parameters of each myocardial strain analysis modality

\begin{tabular}{|c|c|c|c|}
\hline & CMR tagging & CMR feature tracking & STE \\
\hline \multicolumn{4}{|l|}{ Basic strain septum } \\
\hline (1) AVC strain septum (\%) & $2.4 \pm 5.8$ & $-1.1 \pm 5.0$ & $-6.8 \pm 7.1$ \\
\hline (2) Peak strain septum (\%) & $-4.0 \pm 2.8$ & $-5.1 \pm 3.6$ & $-10.4 \pm 5.4$ \\
\hline (3) $\mathrm{TTP}_{\max }$ septum (ms) & $195 \pm 179$ & $379 \pm 211$ & $459 \pm 173$ \\
\hline (4) Systolic strain rate septum (\%/s) & $5.4 \pm 16.4$ & $-3.0 \pm 12.9$ & $-15.9 \pm 16.1$ \\
\hline (5) Diastolic strain rate septum $(\% / s)$ & $-1.1 \pm 10.8$ & $2.1 \pm 10.9$ & $-1.9 \pm 29.0$ \\
\hline \multicolumn{4}{|l|}{ Basic strain lateral wall } \\
\hline (1) AVC strain lateral (\%) & $-12.6 \pm 3.2$ & $-12.0 \pm 3.5$ & $-14.5 \pm 5.3$ \\
\hline (2) Peak strain lateral (\%) & $-13.4 \pm 2.7$ & $-12.4 \pm 3.6$ & $-15.8 \pm 5.5$ \\
\hline (3) $\mathrm{TTP}_{\max }$ lateral (ms) & $424 \pm 33$ & $404 \pm 31$ & $474 \pm 52$ \\
\hline (4) Systolic strain rate lateral (\%/s) & $-32.2 \pm 7.9$ & $-30.8 \pm 9.3$ & $-32.8 \pm 11.9$ \\
\hline (5) Diastolic strain rate lateral (\%/s) & $29.7 \pm 13.2$ & $27.8 \pm 13.7$ & $12.5 \pm 25.0$ \\
\hline \multicolumn{4}{|l|}{ Dyssynchrony } \\
\hline (a) Onset-delay (ms) & $55 \pm 25$ & $58 \pm 46$ & $57 \pm 61$ \\
\hline (b) Peak-delay (ms) & $268 \pm 127$ & $189 \pm 104$ & $144 \pm 104$ \\
\hline (c) $\mathrm{TTP}_{\mathrm{SD}}(\mathrm{ms})$ & $149 \pm 48$ & $159 \pm 44$ & $149 \pm 52$ \\
\hline \multicolumn{4}{|l|}{ Discoordination septal and lateral wall } \\
\hline (d) $\mathrm{SRS}_{\text {sept }}(\%)$ & $7.2 \pm 4.5$ & $3.8 \pm 2.6$ & $3.6 \pm 3.9$ \\
\hline (e) SSI (\%) & $8.7 \pm 5.5$ & $5.1 \pm 3.8$ & $5.0 \pm 4.4$ \\
\hline (f) $\mathrm{ISF}_{\text {sep-lat }}$ & $0.43 \pm 0.25$ & $0.29 \pm 0.21$ & $0.25 \pm 0.16$ \\
\hline \multicolumn{4}{|l|}{ (g) Septal strain patterns $(\mathrm{n}, \%)$} \\
\hline LBBB-1 & $7(26)$ & $5(19)$ & $10(37)$ \\
\hline LBBB-2 & $15(56)$ & $13(48)$ & $6(22)$ \\
\hline LBBB-3 & $5(19)$ & $9(33)$ & $11(41)$ \\
\hline \multicolumn{4}{|l|}{ Discoordination total LV } \\
\hline (h) $\mathrm{ISF}_{\mathrm{LV}}$ & $0.46 \pm 0.22$ & $0.35 \pm 0.17$ & $0.37 \pm 0.13$ \\
\hline (i) CURE & $0.81 \pm 0.09$ & $0.77 \pm 0.09$ & $0.78 \pm 0.06$ \\
\hline
\end{tabular}

CMR cardiac magnetic resonance imaging, TAG tagging, FT feature tracking, STE speckle tracking echocardiography, AVC strain strain value at aortic valve closure, $T T P_{\max }$ time to maximal peak shortening, onset-delay time delay between onset of shortening of septal and lateral wall, peak-delay septal to lateral wall delay of $\mathrm{TTP}_{\max }, T T P_{S D}$ standard deviation if time to peak max of all segments, $S R S_{\text {sept }}$ septal systolic rebound stretch, $S S I$ systolic stretch index, $I S F_{\text {sep-lat }}$ internal stretch factor of septum and lateral wall, $I S F_{L V}$ internal stretch factor of all left ventricular segments, CURE circumferential uniformity ratio estimates
CMR-TAG versus CMR-FT. Nevertheless, echocardiography has its known limitations. High quality images are required for reliable strain analysis with STE, $[29,30]$ but can be difficult in this selection of patients. Frame rate is directly related to the temporal resolution, which is often high in echocardiographic images, especially compared to the relative low frame rate of standard cine images used for CMR-FT. A low frame rate causes under sampling and may lead to misinterpretation of peak and time-to-peak values in strain signals [29]. The frame rate of CMR-TAG was relatively high and comparable to STE in our study. Therefore, CMR-TAG may be considered a true gold-standard technique in this study, as imaging quality and frame rate of the implemented tagging protocol were optimized.

\section{Assessment of strain parameters}

Peak strain parameters showed fair correlation, especially between CMR techniques, except for timing indices of the septum. The maximal peak of septal strain can shift easily in case of dyssynchrony, as there are often multiple peaks (e.g. LBBB-1 and LBBB-2 patterns). Changes in absolute strain values of these peaks can drastically change $\mathrm{TTP}_{\max }$. In previous studies, most dyssynchrony and discoordination parameters have been primarily analysed with a single imaging technique. While some (i.e. CURE and ISF $_{\mathrm{LV}}$ ) are predominantly used in CMR-based studies,[20] others (i.e. SRS $_{\text {sept }}$, peak-delay and septal strain patterns) are primarily derived with STE [12]. In our study, basic strain parameters, and more complex parameters of mechanical dyssynchrony showed apparent variations among the three techniques. However, the three techniques did show fair 
CMR-TAG vs. CMR-FT
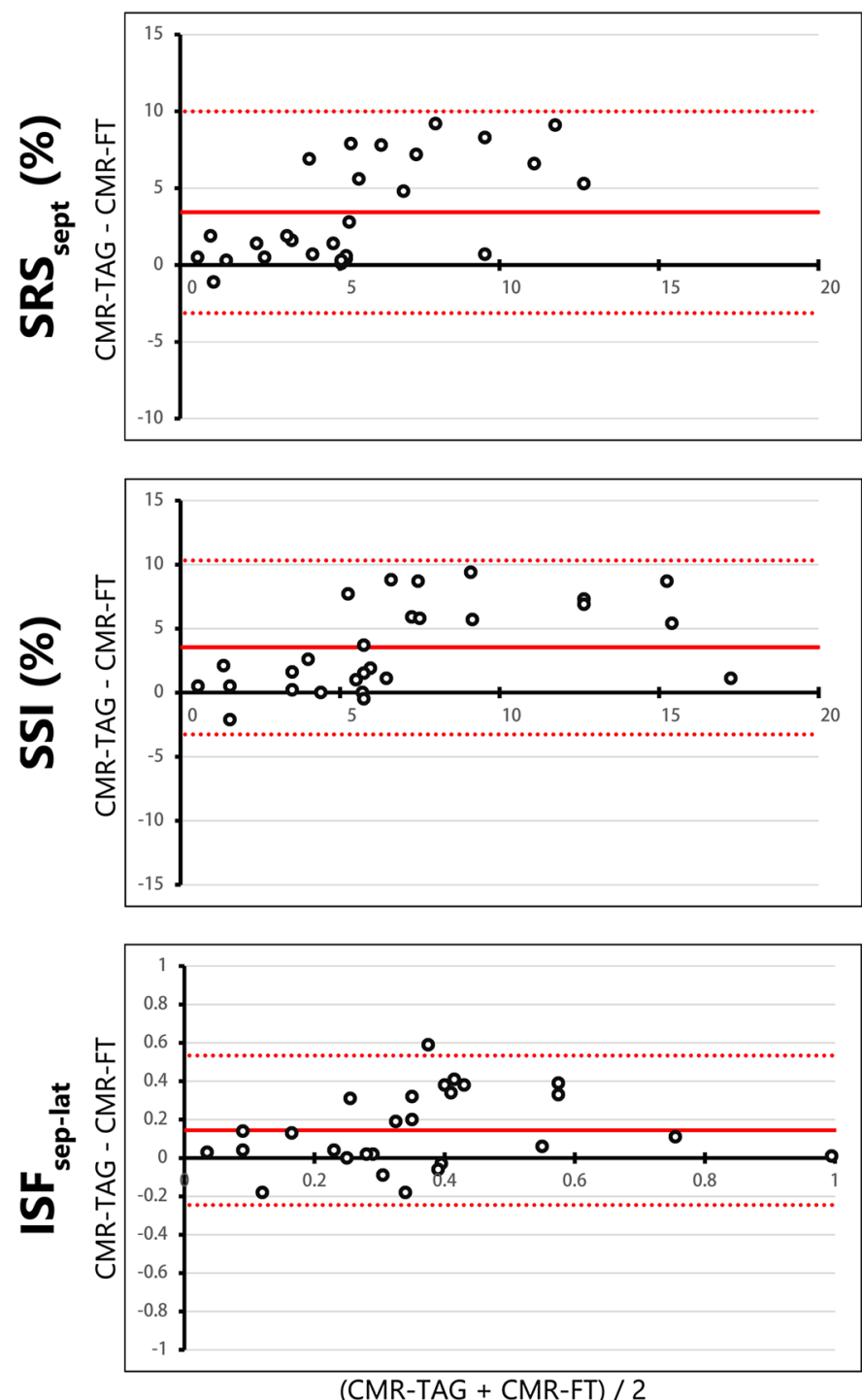

Fig. 4 Bland-Altman plots of regional discoordination parameters. Bland-Altman plots for CMR-TAG versus CMR-FT and CMR-TAG versus STE of regional discoordination parameters (i.e. SRS $_{\text {sept }}$, SSI and ISF $\left._{\text {sep-lat }}\right)$. The mean value of one patient analysed with the two techniques is plotted on the $\mathrm{x}$-axis and the difference on the $\mathrm{y}$-axis. The mean difference is displayed as a solid red line, while the limits

agreement on discoordination parameters. This indicates that these parameters adequately reflect mechanical discoordination and that they are detectable by multiple modalities. Discoordination parameters are promising as predictors for CRT response $[10,11,20]$. The predictive value of discoordination parameters is even known in combination with electrocardiographic parameters [6,11]. $\mathrm{ISF}_{\mathrm{LV}}$ and CURE are predictors of CRT response and use information of all available LV segments, [31] therefore reflecting total LV discoordination [20,21]. These parameters are also less susceptible to outliers compared to basic strain parameters, as they
CMR-TAG vs. STE
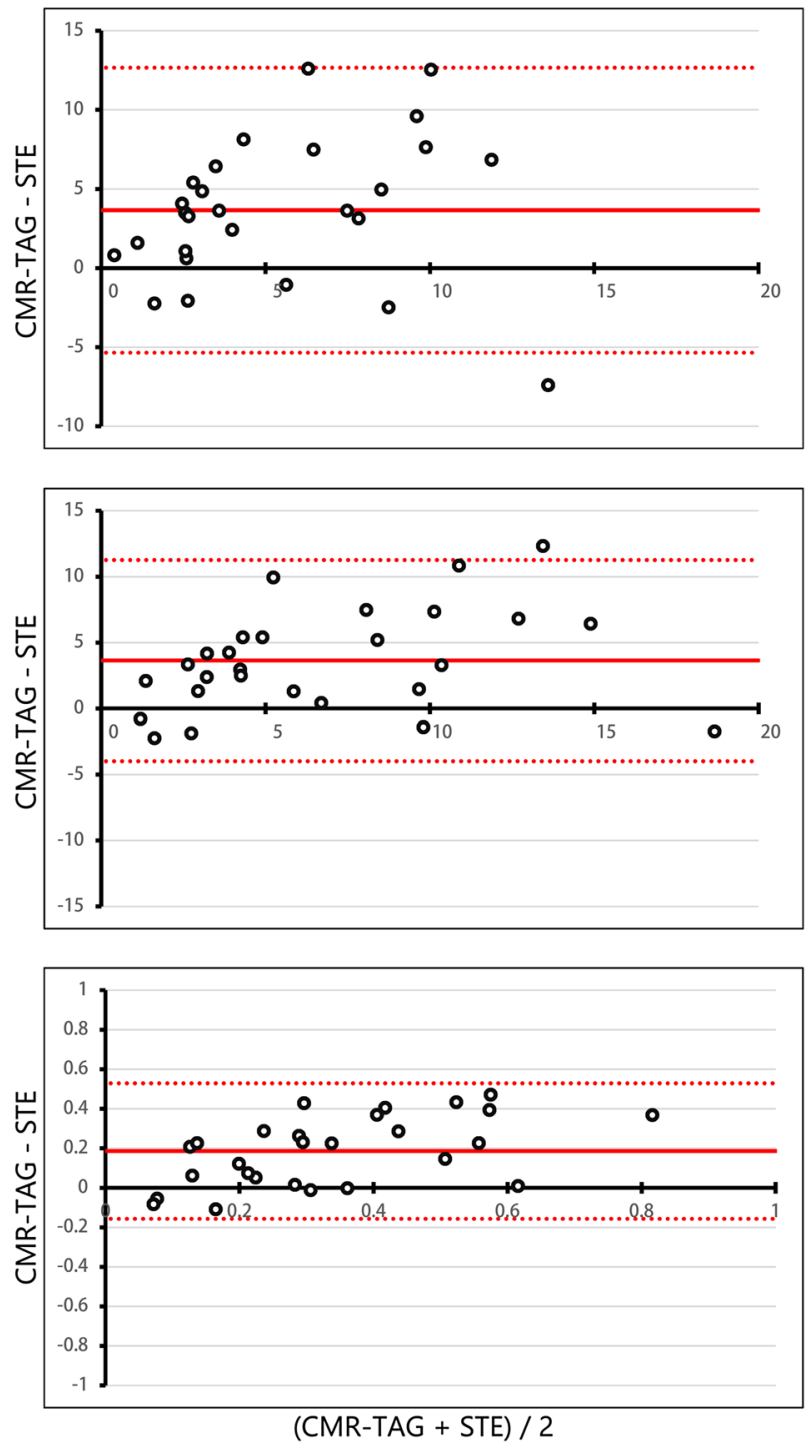

of agreement are displayed as dotted red lines. $S R S_{\text {sept }}$ septal systolic rebound stretch, SSI systolic stretch index, $I S F_{\text {sep-lat }}$ internal stretch factor of septum and lateral wall, $C M R$ cardiac magnetic resonance imaging, TAG tagging, FT feature tracking, STE speckle tracking echocardiography

contain information on all segments [20,21]. Parameters being calculated using averages of multiple segments (i.e. $\mathrm{SRS}_{\text {sept }}$, SSI) also showed fair agreement between modalities. Obtaining deformation characteristics using averages of multiple segments may therefore reduce noise and measurement variability. Specific pre-specified septal strain patterns are known to predict CRT response, as LBBB-1 and LBBB-2 patterns are associated with volumetric response after CRT, while LBBB-3 is not $[12,32]$. The relative high agreement between CMR-TAG and CMR-FT on LBBB-1 and LBBB- 2 on the one hand, and LBBB- 3 on the other 
CMR-TAG vS. CMR-FT
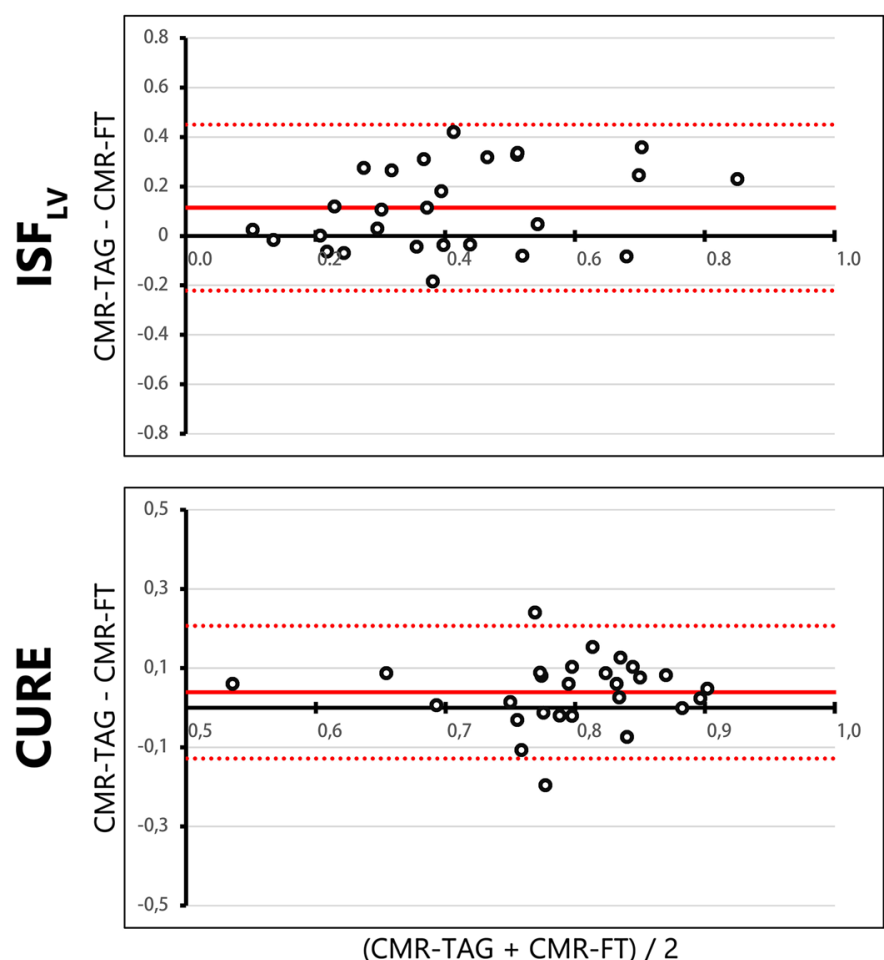

$(\mathrm{CMR}-\mathrm{TAG}+\mathrm{CMR}-\mathrm{FT}) / 2$

Fig. 5 Bland-Altman plots of discoordination parameters of the total LV. Bland-Altman plots for CMR-TAG versus CMR-FT and CMRTAG versus STE of two discoordination parameters, obtained from the total LV. The mean value of one patient analysed with the two techniques is plotted on the $\mathrm{x}$-axis and the difference on the $\mathrm{y}$-axis. The mean difference is displayed as a solid red line, while the limits

is therefore promising for further implementation of septal strain pattern categorization using CMR.

\section{Myocardial strain orientation}

STE parameters are mainly validated with longitudinal strain, [33, 34] while CMR is predominantly based on circumferential strain [20,21]. Circumferential strain is more intuitive, as mid-myocardial fibres are orientated in the circumferential direction and short-axis images represent all segments distributed around the LV at each level (i.e. basal, mid or apical) [35]. The method of determining circumferential strain calculation differs between the three methods. Both the CMR-FT and STE software track specific myocardial details, respectively 'features' and 'speckles', of the endo- and epicardial border [14]. The specific wall layer used for strain analysis differed between techniques. The results of the endocardial layer were used for STE, as the epicardial layer often lacked an appropriate border zone. Strain values of CMR-FT were a product of endocardial and epicardial strain. This is in contrast to CMR-TAG, of which strain of the mid-wall layer was used

\section{CMR-TAG vs. STE}
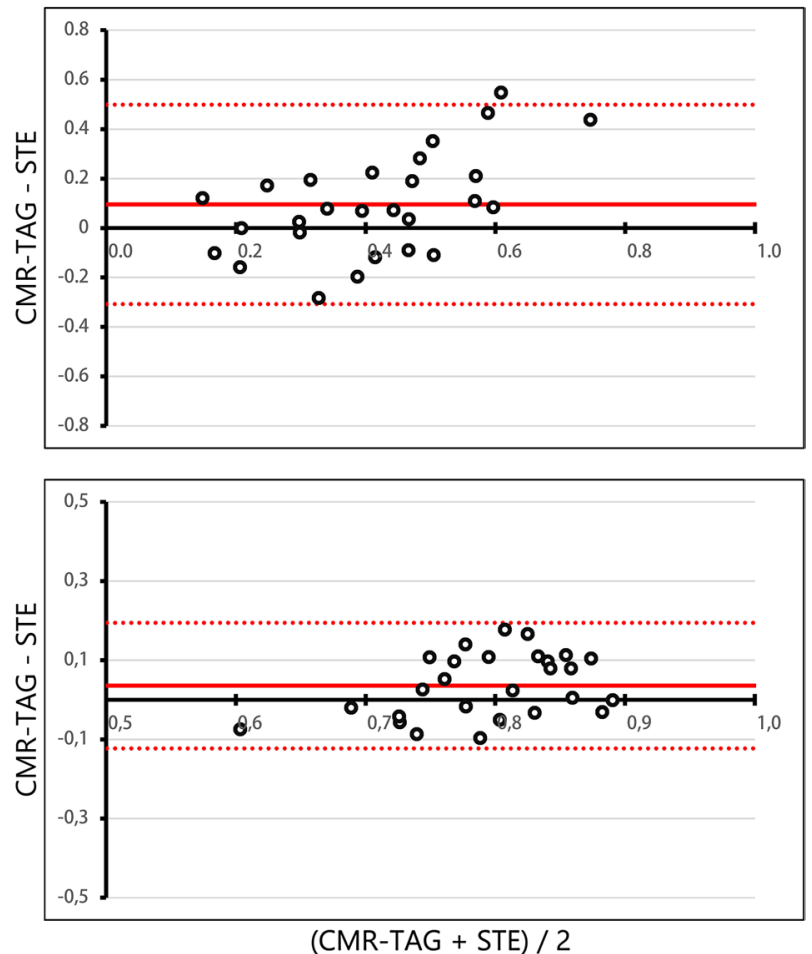

of agreement are displayed as dotted red lines. $I S F_{L V}$ internal stretch factor of the total LV, CURE circumferential uniformity ratio estimates, $L V$ left ventricle, $C M R$ cardiac magnetic resonance imaging, $T A G$ tagging, $F T$ feature tracking, $S T E$ speckle tracking echocardiography

[19]. The difference between the approaches may have biased the overall level of agreement. Endocardial strain is known to give higher peak values compared to epicardial strain [36], and might also be higher than midmyocardial values, which can be appreciated in the positive mean difference between CMR-TAG and STE on peak strain and AVC-strain in the Bland-Altman results. This difference may have also affected the agreement of dyssynchrony and discoordination parameters.

\section{Limitations}

CMR imaging with myocardial tagging was performed in a small subset of patients from the MARC study, which may have given outliers a relatively large effect on results. The patient population was moreover limited to patients eligible for CRT, reducing variability in measurements. These results should therefore be validated in a larger cohort. However, strain measurements are of particular interest in this specific population to improve patient selection for CRT. As mentioned, the study protocol has also influenced 
Table 3 Intra-class correlation and correlation of CMR tagging versus CMR feature tracking and CMR tagging versus speckle tracking echocardiography

\begin{tabular}{|c|c|c|c|c|}
\hline & \multicolumn{2}{|c|}{$\begin{array}{l}\text { CMR-TAG vs. CMR-FT } \\
(\mathrm{n}=27)\end{array}$} & \multicolumn{2}{|c|}{ CMR-TAG vs. STE $(n=27)$} \\
\hline & $\operatorname{ICC}(95 \% \mathrm{CI})$ & $\mathrm{R}$ & $\operatorname{ICC}(95 \% \mathrm{CI})$ & $\mathrm{R}$ \\
\hline \multicolumn{5}{|l|}{ Basic strain septum } \\
\hline (1) AVC strain septum (\%) & $0.55(0.09-0.79)$ & $0.67^{\ddagger}$ & $0.23(-0.10-0.56)$ & $0.47 *$ \\
\hline (2) Peak strain septum (\%) & $0.58(0.26-0.78)$ & $0.55^{\dagger}$ & $0.155(-0.10-0.45)$ & $0.42 *$ \\
\hline (3) $\mathrm{TTP}_{\max }$ septum (ms) & $0.17(-0.11-0.47)$ & 0.11 & $0.00(-0.15-0.22)$ & -0.16 \\
\hline (4) Systolic strain rate septum (\%/s) & $0.56(0.16-0.79)$ & $0.66^{\ddagger}$ & $0.25(-0.10-0.57)$ & $0.45^{*}$ \\
\hline (5) Diastolic strain rate septum $(\% / s)$ & $0.64(0.35-0.82)$ & $0.66^{\ddagger}$ & $0.34(-0.05-0.635)$ & $0.50^{\dagger}$ \\
\hline \multicolumn{5}{|l|}{ Basic strain lateral wall } \\
\hline (1) AVC strain lateral (\%) & $0.50(0.16-0.74)$ & $0.50^{\dagger}$ & $0.08(-0.27-0.43)$ & 0.10 \\
\hline (2) Peak strain lateral (\%) & $0.54(0.22-0.76)$ & $0.59^{\dagger}$ & $0.01(-0.31-0.36)$ & 0.02 \\
\hline (3) $\mathrm{TTP}_{\max }$ lateral (ms) & $0.34(0.00-0.63)$ & $0.40 *$ & $0.13(-0.12-0.41)$ & 0.23 \\
\hline (4) Systolic strain rate lateral (\%/s) & $0.575(0.26-0.78)$ & $0.58^{\dagger}$ & $0.05(-0.35-0.42)$ & 0.05 \\
\hline (5) Diastolic strain rate lateral (\%/s) & $0.82(0.65-0.91)$ & $0.82^{\ddagger}$ & $0.23(-0.08-0.53)$ & 0.38 \\
\hline \multicolumn{5}{|l|}{ Dyssynchrony } \\
\hline (a) Onset-delay (ms) & $0.42(0.05-0.69)$ & 0.23 & $0.024(-0.37-0.40)$ & -0.08 \\
\hline (b) Peak-delay (ms) & $0.45(0.045-0.715)$ & $0.46^{*}$ & $0.23(-0.09-0.53)$ & 0.27 \\
\hline (c) $\mathrm{TTP}_{\mathrm{SD}}(\mathrm{ms})$ & $0.46(0.11-0.71)$ & $0.49^{\dagger}$ & $0.20(-0.20-0.54)$ & 0.19 \\
\hline \multicolumn{5}{|l|}{ Regional discoordination } \\
\hline (d) $\mathrm{SRS}_{\text {sept }}(\%)$ & $0.41(-0.06-0.72)$ & $0.65^{\text {市}}$ & $0.30(-0.05-0.60)$ & $0.41 *$ \\
\hline (e) SSI (\%) & $0.58(0.00-0.83)$ & $0.68^{\ddagger}$ & $0.55(0.02-0.81)$ & $0.70^{\ddagger}$ \\
\hline (f) $\mathrm{ISF}_{\text {sep-lat }}$ & $0.53(0.10-0.77)$ & $0.45^{*}$ & $0.46(-0.06-0.76)$ & $0.69^{\ddagger}$ \\
\hline \multicolumn{5}{|l|}{ Discoordination total LV } \\
\hline (h) $\mathrm{ISF}_{\mathrm{LV}}$ & $0.55(0.15-0.78)$ & $0.66^{\ddagger}$ & $0.32(-0.02-0.61)$ & $0.42 *$ \\
\hline (i) CURE & $0.485(0.145-0.725)$ & 0.37 & $0.41(0.06-0.67)$ & 0.36 \\
\hline
\end{tabular}

$C I$ confidence interval, $I C C$ intra-class correlation coefficient, $R$ correlation coefficient, for other abbreviations see Table 2

$\mathrm{P}$ values for statistical significance of $\mathrm{R}$-values are given with: ${ }^{*} \mathrm{p}$ value $<0.05,{ }^{\dagger} \mathrm{p}$ value $<0.01,{ }^{\star} \mathrm{p}$ value $<0.001$ results, as echocardiographic and CMR examination were not performed on the same day. Moreover, differences in imaging plane between CMR and STE are possible and strain analysis was not performed on the same wall layers. ECG triggering differs between imaging techniques, as ECG electrodes were repositioned between examinations and a different lead may have been used. Moreover, ECG triggering of STE was placed at QRS onset, while the top of the $\mathrm{R}$ wave is used for CMR. ECG triggering affects the reference value and may have affected subsequent values of timing and absolute changes. While STE relied on end-diastolic region of interest placement, CMR-FT used both end-systolic and end-diastolic region of interests to determine myocardial strain. The reliability of CMR-FT may therefore be higher. Echocardiography was moreover obtained with ultrasound machines from two vendors, possibly introducing differences in source data. The overall lower agreement of CMR-TAG and STE should therefore be appreciated carefully. STE was performed with circumferential strain obtained from short axis images, for a more direct comparison between techniques. While circumferential strain is widely used in scientific publications, standardization of algorithms of STE has also mainly been done for longitudinal strain [5, 34]. Longitudinal strain assessed with STE may therefore have a higher reliability and reproducibility compared to circumferential strain. The effect of using longitudinal or circumferential strain derived with STE for prediction of CRT response deserves attention in future work.

\section{Clinical application}

The overall reasonable agreement between CMR-TAG and CMR-FT is promising for clinical application. CMR-FT might be a reasonable alternative for CMR-TAG and STE, as suitable CMR cine images are more easily available in clinical practice, compared to the highly specialized CMR-TAG protocols. Detection of mechanical discoordination with CMR-FT is a valuable addition to CMR, 
which already constitutes an important imaging tool in CRT-candidates for accurate determination of the LV ejection fraction and scar tissue localization [31]. On the other hand, a portable and bed-side tool like STE might have the highest clinical applicability, of which most discoordination parameters also showed fair agreement compared to the CMR-TAG. The reasonable agreement of the three techniques on mechanical discoordination parameters is moreover promising for the prediction of response to CRT. The implemented discoordination parameters were previously associated with CRT response in single centre studies $[10,11,20]$. However, previous markers of CRT response failed to take the final step to clinical application, partly because validation to gold-standard techniques was missing [9]. As the specific methods and modality may slightly differ from previous publications, further studies are needed for implementation into clinical practice. Future studies will focus on the predictive value of these parameters using follow-up data in this specific population.

\section{Conclusions}

In conclusion, comparison of strain analysis techniques showed that CMR-FT had an overall fair agreement with gold-standard CMR-TAG. Although agreement between STE and CMR-TAG was overall lower, direct comparison was limited by technical and methodological differences. The agreement was highest for parameters of mechanical discoordination, compared to basic strain or dyssynchrony parameters. CMR-FT is therefore a potentially valuable clinical alternative for CMR-TAG and STE, especially in the evaluation of mechanical discoordination in CRT-candidates.

Funding This research was funded within the framework of CTMM, the Centre for Translational Molecular Medicine (http://www.ctmm. nl), project COHFAR (Grant 01C-203), and supported by the Dutch Heart Foundation.

Authors' contributions WE and MC analyzed and interpreted the patient data regarding the echocardiograms and speckle tracking analysis. AZ and RN analyzed and interpreted the patient data regarding the cardiac magnetic resonance imaging, including CMR feature tracking and tagging. $\mathrm{AM}, \mathrm{KV}, \mathrm{MM}, \mathrm{FL}$ and $\mathrm{CA}$ were local principal investigators. IG and MV were central principal investigators. PC and PC assisted with the CMR tagging software. BG and MR performed the statistical analyses. WE, AZ, RN, MC, and CA were a major contributor in writing the manuscript. All authors read and approved the final manuscript.

\section{Compliance with ethical standards}

Conflict of interests Wouter M. van Everdingen, Alwin Zweerink, Robin Nijveldt, Odette A.E. Salden, Mathias Meine, Alexander H.
Maass, Frederik J. De Lange, Albert C. van Rossum, Pierre Croisille, Patrick Clarysse, Bastiaan Geelhoed, Michiel Rienstra, Isabelle C. Van Gelder, Cornelis P. Allaart, Maarten J. Cramer declare that they have no conflict of interests. Dr. Vernooy received consultancy fee from Medtronic; research grants from Medtronic; speaker fees from St. Jude Medical. Dr. Maass received lecture fees from Medtronic and LivaNova. Dr. Vos received funding from CTMM COHFAR, CVON Predict, EU TrigTreat, EU CERT-ICD and GiLead to perform (pre) clinical studies.

Ethical approval All procedures performed in studies involving human participants were in accordance with the ethical standards of the institutional and/or national research committee and with the 1964 Helsinki declaration and its later amendments or comparable ethical standards.

Informed consent Informed consent was obtained from all individual participants included in the study.

Open Access This article is distributed under the terms of the Creative Commons Attribution 4.0 International License (http://creativecommons.org/licenses/by/4.0/), which permits unrestricted use, distribution, and reproduction in any medium, provided you give appropriate credit to the original author(s) and the source, provide a link to the Creative Commons license, and indicate if changes were made.

\section{References}

1. Brignole M, Auricchio A, Baron-Esquivias G, Bordachar P, Boriani G, Breithardt OA et al (2013) 2013 ESC Guidelines on cardiac pacing and cardiac resynchronization therapy. Eur Heart $\mathbf{J}$ 34(29):2281-2329

2. Sweeney MO, Prinzen FW (2008) Ventricular pump function and pacing: physiological and clinical integration. Circ Arrhythm Electrophysiol 1(2):127-139

3. Daubert JC, Saxon L, Adamson PB, Auricchio A, Berger RD, Beshai JF et al (2012) 2012 EHRA/HRS expert consensus statement on cardiac resynchronization therapy in heart failure: implant and follow-up recommendations and management. Europace 14(9):1236-1286

4. Auricchio A, Prinzen FW (2011) Non-responders to cardiac resynchronization therapy: the magnitude of the problem and the issues. Circ J 75(3):521-527

5. van Everdingen WM, Schipper JC, van 't Sant J, Ramdat Misier K, Meine M, Cramer MJ (2016) Echocardiography and cardiac resynchronisation therapy, friends or foes? Netherlands Heart $\mathbf{J}$ 24(1):25-38

6. Risum N, Tayal B, Hansen TF, Bruun NE, Jensen MT, Lauridsen TK et al (2015) Identification of typical left bundle branch block contraction by strain echocardiography is additive to electrocardiography in prediction of long-term outcome after cardiac resynchronization therapy. J Am Coll Cardiol 66(6):631-641

7. Wang CL, Wu CT, Yeh YH, Wu LS, Chan YH, Kuo CT et al (2017) Left bundle-branch block contraction patterns identified from radial-strain analysis predicts outcomes following cardiac resynchronization therapy. Int J Cardiovasc Imaging 33(6):869-877

8. Leenders GE, Cramer MJ, Bogaard MD, Meine M, Doevendans PA, De Boeck BW (2011) Echocardiographic prediction of outcome after cardiac resynchronization therapy: conventional methods and recent developments. Heart Fail Rev 16(3):235-250 
9. Chung ES, Leon AR, Tavazzi L, Sun JP, Nihoyannopoulos P, Merlino J et al (2008) Results of the Predictors of Response to CRT (PROSPECT) trial. Circulation 117(20):2608-2616

10. Leenders GE, De Boeck BW, Teske AJ, Meine M, Bogaard MD, Prinzen FW et al (2012) Septal rebound stretch is a strong predictor of outcome after cardiac resynchronization therapy. J Card Fail 18(5):404-412

11. Lumens J, Tayal B, Walmsley J, Delgado-Montero A, Huntjens PR, Schwartzman D et al (2015) Differentiating electromechanical from non-electrical substrates of mechanical discoordination to identify responders to cardiac resynchronization therapy. Circ Cardiovasc Imaging 8(9):e003744

12. Leenders GE, Lumens J, Cramer MJ, De Boeck BW, Doevendans PA, Delhaas T et al (2012) Septal deformation patterns delineate mechanical dyssynchrony and regional differences in contractility: analysis of patient data using a computer model. Circ Heart Fail 5(1):87-96

13. Revah G, Wu V, Huntjens PR, Piekarski E, Chyou JY, Axel L (2016) Cardiovascular magnetic resonance features of mechanical dyssynchrony in patients with left bundle branch block. Int J Cardiovasc Imaging 32(9):1427-1438

14. Pedrizzetti G, Claus P, Kilner PJ, Nagel E (2016) Principles of cardiovascular magnetic resonance feature tracking and echocardiographic speckle tracking for informed clinical use. J Cardiovasc Magn Reson 18(1):51

15. Harrild DM, Han Y, Geva T, Zhou J, Marcus E, Powell AJ (2012) Comparison of cardiac MRI tissue tracking and myocardial tagging for assessment of regional ventricular strain. Int J Cardiovasc Imaging 28(8):2009-2018

16. Zwanenburg JJ, Gotte MJ, Kuijer JP, Heethaar RM, van Rossum AC, Marcus JT (2004) Timing of cardiac contraction in humans mapped by high-temporal-resolution MRI tagging: early onset and late peak of shortening in lateral wall. Am J Physiol Heart Circ Physiol 286(5):H1872-H1880

17. Maass AH, Vernooy K, Wijers SC, van 't Sant J, Cramer MJ, Meine $\mathrm{M}$ et al. (2017) Refining success of cardiac resynchronization therapy using a simple score predicting the amount of reverse ventricular remodelling: results from the Markers and Response to CRT (MARC) study. Europace. doi:10.1093/europace/euw445

18. Lang RM, Badano LP, Mor-Avi V, Afilalo J, Armstrong A, Ernande L et al (2015) Recommendations for cardiac chamber quantification by echocardiography in adults: an update from the American Society of Echocardiography and the European Association of Cardiovascular Imaging. Eur Heart J Cardiovasc Imaging 16(3):233-270

19. Miller CA, Borg A, Clark D, Steadman CD, McCann GP, Clarysse P et al (2013) Comparison of local sine wave modeling with harmonic phase analysis for the assessment of myocardial strain. J Magn Reson Imaging 38(2):320-328

20. Kirn B, Jansen A, Bracke F, van Gelder B, Arts T, Prinzen FW (2008) Mechanical discoordination rather than dyssynchrony predicts reverse remodeling upon cardiac resynchronization. Am J Physiol Heart Circ Physiol 295(2):H640-H646

21. Helm RH, Leclercq C, Faris OP, Ozturk C, McVeigh E, Lardo $\mathrm{AC}$ et al (2005) Cardiac dyssynchrony analysis using circumferential versus longitudinal strain: implications for assessing cardiac resynchronization. Circulation 111(21):2760-2767

22. Shrout PE, Fleiss JL (1979) Intraclass correlations: uses in assessing rater reliability. Psychol Bull 86(2):420-428

23. Castillo E, Osman NF, Rosen BD, El-Shehaby I, Pan L, Jerosch-Herold M et al (2005) Quantitative assessment of regional myocardial function with MR-tagging in a multi-center study: interobserver and intraobserver agreement of fast strain analysis with Harmonic Phase (HARP) MRI. J Cardiovasc Magn Reson 7(5):783-791

24. van Everdingen WM, Paiman ML, van Deursen CJ, Cramer MJ, Vernooy K, Delhaas T et al (2015) Comparison of septal strain patterns in dyssynchronous heart failure between speckle tracking echocardiography vendor systems. J Electrocardiol 48(4):609-616

25. Wu L, Germans T, Guclu A, Heymans MW, Allaart CP, van Rossum AC (2014) Feature tracking compared with tissue tagging measurements of segmental strain by cardiovascular magnetic resonance. J Cardiovasc Magn Reson 16:10

26. Onishi T, Saha SK, Ludwig DR, Marek JJ, Cavalcante JL, Schelbert EB et al (2013) Feature tracking measurement of dyssynchrony from cardiovascular magnetic resonance cine acquisitions: comparison with echocardiographic speckle tracking. J Cardiovasc Magn Reson 15:95

27. Moody WE, Taylor RJ, Edwards NC, Chue CD, Umar F, Taylor TJ et al (2015) Comparison of magnetic resonance feature tracking for systolic and diastolic strain and strain rate calculation with spatial modulation of magnetization imaging analysis. J Magn Reson Imaging 41(4):1000-1012

28. Singh A, Steadman CD, Khan JN, Horsfield MA, Bekele S, Nazir SA et al (2015) Intertechnique agreement and interstudy reproducibility of strain and diastolic strain rate at 1.5 and $3 \mathrm{~T}$ : a comparison of feature-tracking and tagging in patients with aortic stenosis. J Magn Reson Imaging 41(4):1129-1137

29. Rosner A, Barbosa D, Aarsaether E, Kjonas D, Schirmer H, D'Hooge J (2015) The influence of frame rate on two-dimensional speckle-tracking strain measurements: a study on silico-simulated models and images recorded in patients. Eur Heart J Cardiovasc Imaging 16(10):1137-1147

30. D'Hooge J, Barbosa D, Gao H, Claus P, Prater D, Hamilton J et al (2016) Two-dimensional speckle tracking echocardiography: standardization efforts based on synthetic ultrasound data. Eur Heart J Cardiovasc Imaging 17(6):693-701

31. Bilchick KC, Dimaano V, Wu KC, Helm RH, Weiss RG, Lima JA et al (2008) Cardiac magnetic resonance assessment of dyssynchrony and myocardial scar predicts function class improvement following cardiac resynchronization therapy. J Am Coll Cardiol Img 1(5):561-568

32. Marechaux S, Guiot A, Castel AL, Guyomar Y, Semichon M, Delelis F et al (2014) Relationship between two-dimensional speckle-tracking septal strain and response to cardiac resynchronization therapy in patients with left ventricular dysfunction and left bundle branch block: a prospective pilot study. J Am Soc Echocardiogr 27(5):501-511

33. De Boeck BW, Teske AJ, Meine M, Leenders GE, Cramer MJ, Prinzen FW et al (2009) Septal rebound stretch reflects the functional substrate to cardiac resynchronization therapy and predicts volumetric and neurohormonal response. Eur J Heart Fail 11(9):863-871

34. Farsalinos KE, Daraban AM, Unlu S, Thomas JD, Badano LP, Voigt JU (2015) Head-to-head comparison of global longitudinal strain measurements among nine different vendors: the EACVI/ ASE Inter-Vendor Comparison Study. J Am Soc Echocardiogr 28(10):1171-1181, e1172

35. de Simone G, Ganau A, Roman MJ, Devereux RB (1997) Relation of left ventricular longitudinal and circumferential shortening to ejection fraction in the presence or in the absence of mild hypertension. J Hypertens 15(9):1011-1017

36. Leitman M, Lysiansky M, Lysyansky P, Friedman Z, Tyomkin V, Fuchs $T$ et al (2010) Circumferential and longitudinal strain in 3 myocardial layers in normal subjects and in patients with regional left ventricular dysfunction. J Am Soc Echocardiogr 23(1):64-70 